\title{
Executive Functioning and Problem Solving: A Bidirectional Relation
}

\author{
https://doi.org/10.3991/ijep.v9i3.10186 \\ Athanasios Drigas $\left.{ }^{(}\right)$, Maria Karyotaki \\ N.C.S.R. ,Demokritos', Agia Paraskevi, Athens, Greece \\ dreiit.demokritos.gr
}

\begin{abstract}
There is a bidirectional relation between one's executive functioning abilities and problem solving skills as they are both based on selfcontrol. "Hot" and "cold" executive functions account for individuals' cognitive control and emotional regulation. In addition, problem solving is tied to metacognitive awareness processes, necessary for applying executive function skills in goal setting and decision making situations. Therefore, individuals' overall cognitive flexibility and emotional regulation can promote the quality, quantity and speed of decision-making processes, such as adaptable and creative information processing as well as efficiency in setting and prioritizing goals. Moreover, individuals with ADHD, Autism Spectrum Disorder, Oppositional Defiant Disorder and individuals with other comorbid states, such as older adults, individuals with Traumatic Brain Injury (TBI) can counterbalance their cognitive control deficits through enhancing their problem solving skills. In addition, an advanced research in the bidirectional relation between executive function and problem solving skills could develop a comprehensive methodology for training and assessing self-regulatory processes.
\end{abstract}

Keywords - Attentional processes, cognitive control, cognitive flexibility, emotional regulation

\section{Introduction}

"The definition of executive function usually includes the concept of controlling attention, mental flexibility, goal-directed behavior and the ability to anticipate the consequences of one's own behavior. Moreover, the concept of self-awareness and the idea that the frontal lobes serve as a manager and programmer of human psychological processes (metacognition) are also included in this definition" (Ardila, 2016)[1].

According to Ardila[2], there are two distinct subgroups constituting individuals' executive functioning; Metacognitive as well as Emotional/Motivational executive functions. Metacognitive executive functions refer to response inhibition, conflict monitoring and switching, self-awareness, temporality of behavior, selfconsciousness, working memory, abstraction and problem solving. 
Emotional/motivational executive functions entail the coordination of cognition and motivation as well as the ability to control emotions and behavior. Also, Jiang et al.[23] discriminated individuals' executive functions in "cold" and "hot". Petrovic et al.[22] initiated an integrated model of emotional and non-emotional, related networks with hierarchical perception-action organization tied to complex and temporally dispersed, information processing. Channon [20] referred to the independence of social cognition and non-social executive abilities.

By investigating the relation between one's cognitive/metacognitive skills and selfconscious emotions, individuals' executive functioning could be substantially improved. Thus, the current research aims to illustrate the common neuropsychological background of learning and self-development. Furthermore, problem-solving skills have a major role in one's everyday life and their significance led to their inclusion in educational and therapeutic settings as a higher-order mental ability for learning and self-improvement purposes [50, 51, 52].

\section{Cognitive and Metacognitive Executive Functions}

Fischer et al. [3] presented the basis of a consistent training and assessment model encompassing Knowledge, Skills, Abilities and Other components (KSAO) regarding the Complex Problem Solving (CPS) competency. Problem solving is necessary for dealing effectively with complex non-routine situations in different domains, thus its requirements in knowledge, skills and abilities are innately dynamic and interrelated. By further exploring the KSAO model in a range of complex problem situations, an innovative methodology for training and assessing the Complex Problem Solving competency shall rise. More specifically, it might be more realistic to investigate a specific set of KSAO components, such as working memory, reasoning ability and intelligence, corresponding to a specific complex problem solving field. It is noteworthy mentioning that several moderating variables, such as problem features and additional factors, such as self-regulation interfere with predicting humans' problem solving performance. Furthermore, when a person solves a problem, by no means, is this indicative of that person's ability to solve another heterogeneous problem.

Rhodes et al. [4] investigated both the direct and indirect relation between executive functions and individuals' memory accuracy in terms of the latter's capacity to recall and monitor correct information. As executive functions decline with age, older adults exhibit low performance both in measures of their memory accuracy and memory monitoring. More specifically, older adults' ability to encode and retrieve information as well as their ability to monitor their feeling-of-knowing judgments diminishes, respectively. Notwithstanding, between executive functions and memory monitoring lays the quality of information available in one's memory. In the current study, there were two recall stages, either the forced recall or the free recall stage. In the forced recall stage participants had to provide as many correct responses as possible and in the free recall stage participants had to control the accuracy of their responses through calling the probability of being correct while setting a response 
criterion. If the probability of being correct exceeds or is equal to the response criterion, an answer is volunteered; otherwise the response is withheld. Control processes influence accuracy by determining whether a response is volunteered or withheld. Therefore, the free recall stage encompasses three criterions in total; Monitoring effectiveness, control sensitivity and the respondents' incentive towards their accuracy estimation. Speed of processing and working memory was separately measured. The measures deployed in the study were: The Digit-Symbol Substitution Task, the Number Comparison Task and the Trail Making Test, the computerized version of the WCST, the Controlled Oral Word Association test and the Working Memory OSPAN task. The findings verified that memory accuracy, quantity of correct items retrieved and executive function were found to be directly related to age. Executive functions affected memory accuracy directly through their contribution to the quantity of correct responses available at the forced recall stage and indirectly through the monitoring of deceptive items at the free recall stage. However, memory monitoring partially mediated the quantity of the correct responses at the free recall stage for deceptive items, but not for control items. Overall, participants' accuracy of retrieved responses and memory monitoring can predict their memory accuracy. Furthermore, the relation between the specific components of executive functions in their full range and memory accuracy measures as well as the possible interplay between retrieval and monitoring processes are yet to be found.

Carden et al.[5] unraveled the role of visualization and working memory in mathematical problem solving performance. Visualization is a strategy in which individuals form a mental image depicting visual or spatial information in a more condensed and flexible manner so as to retain and connect data. The relation between visual-spatial skills and mathematics, including complex problem solving has been certified. The spatial ability and especially, the type of imagery used by individuals, is a significant factor for solving practical problems successfully. In addition, working memory capacity underlies retention and manipulation of information in individuals and it has consistently proven to be an efficient predictor of children's reading skills and mathematical ability. Therefore, visualization strategies and thus one's spatial abilities can mediate individual's working memory performance as he or she seeks for cognitive support when facing novel or complex problem solving situations.

Passolunghi et al.[6] examined the relation between arithmetic problem solving and working memory as well as the relation between arithmetic problem solving and short-term memory. In both cases, the underlying mechanism in the performance of the working memory was expected to be the inhibition of irrelevant information. Therefore, intrusion errors during recall in seven working memory and short-term memory tasks could be linked to the participants' word problem performance and/or to the inherent operation of the working memory, itself. The research was grounded in testing two groups; good problem solvers and poor problem solvers in the Listening span task, the Animal dual task, the Listening span completion task, the Counting span task and the Short-term memory tasks. There seems to be a generalized working memory deficit in poor problem solvers, regardless of verbal or numerical content and regardless of the task difficulty. Furthermore, when the two groups were controlled for their reading comprehension, no significant difference came up. However, the 
poor problem solvers performed worse when the two groups were matched on their verbal intelligence. Thus, both the working memory system and arithmetic problem solving skills as well as one's working memory capacity and inhibition mechanisms seem to be more closely related. Overall, poor problem solvers were found less able to maintain, process or suppress irrelevant information of any kind, verbal and numerical, in their working memory. Metacognition could counterbalance this lack of flexibility in poor problem solvers performance through training their capacity to swift their selective attention to the appropriate strategies for encoding, accessing and inhibiting information from working and long-term memory.

Zheng et al.[7] compared mathematical word problem solving accuracy to elementary school children's working memory components through measuring their problem solving accuracy, problem solving processes, central executive component of working memory, reading and math calculation. Children between 6 and 12 grow faster on their executive ability, although their reading skills, arithmetic proficiency and fluency as well as their acquaintance with word problem solving processes may mediate the relationship between working memory components and problem solving accuracy. Tasks were divided into criterion, predictor and mediator variables. Criterion variables included mental solution of oral word problems. Predictor variables measured the phonological loop, the visual-spatial sketchpad and the central executive system. Mediators were considered measures of arithmetic calculation, reading and knowledge of word problem solving processes. As expected, chronological age was positively related to children's working memory performance as a whole. The tripartite working memory model by Baddeley with each component of working memory contributing unique variance to problem solving accuracy was confirmed in the present study as well. The phonological loop and the visual-spatial sketchpad influenced children's word problem solving accuracy and more specifically, the visual-spatial sketchpad, contributed unique variance to the problem solving accuracy. The central executive component had a prominent role in arithmetic word problem solving due to the controlled attention needed for inhibiting irrelevant information while accessing, updating and integrating information originating from long-term memory. Studies showing a weak correlation between working memory and problem solving were due to children's from 8 to 11 years old overlap between their phonological loop and working memory on several academic measures. As for the word problem solving accuracy, the phonological loop and the central executive were most influenced by academic mediator variables, such as reading and calculation proficiency.

Davidson et al.[8] made a comparative study on children, adolescents and adults' cognitive control skills and executive functions by measuring the interrelations and developmental progressions of their working memory, inhibitory control and task switching skills. Participants were assessed through a battery of 4 related tests for about $30 \mathrm{~min}$ in total. The first test was a classic Simon task, where the stimuli were presented randomly on the left or right of the screen over the block of 20 trials. In the second test, a single large arrow pointed straight down and participants had to respond on the same side as the arrow in a randomized mixed block of 20 congruent and incongruent trials at the left or right of the computer screen. In the Dots test, a large 
dot was presented either at the left or right on each trial. The two types of dots used were either striped or solid, which indicated that when participants saw the striped dot they would have to make a response towards the same side, whereas when they saw the solid dot they would have to respond on the side opposite the dot. An initial block of 20 congruent trials was combined with a block of 20 incongruent trials as well as a block of 20 randomly intermixed trials. In the last test, each stimulus, an abstract shape, was presented in the center of the rectangle. For each stimulus participants were taught a rule and there were two conditions involving two- or six- abstract shapes. They first completed 2 blocks of 20 trials of the two-shape condition accompanied by 2 blocks of 20 trials for the six-shape condition. The dependent measures were accuracy of responses, speed/reaction time (RT) and percentage of anticipatory responses (AR). The results verified researchers' hypothesis that the cost of exercising inhibition to respond faster and more accurately on spatially incompatible trials (the Simon effect) decreased from 6 years of age onward. In regard to the integration of arbitrary rules related to the stimulus of each trial with the location of the stimulus, the spatial incompatibility effect decreased significantly from age 6 onward in accuracy, but only on the Arrows test. On the whole, participants' performance was slower and less accurate on switch than non-switch trials in both the Arrows task and the Dots-Mixed condition. However, the response time did not change between children of 6-13 years and adults on both arrows and Dots-Mixed condition due to the univalent stimuli employed in a task or rule. Furthermore, accuracy and impulsivity differences between the Dots-Incongruent and DotsCongruent conditions decreased over age. Notwithstanding, on both the Arrows test and the Dots-Mixed condition adults and older children showed greater speed response cost for switching to the Congruent rule. Furthermore, adults' response time decreased when both the rule and response-site switched. However, the youngest children of 4-8 years performed better in accuracy and reaction time on switch trials where the response-site remained the same. Therefore, cognitive flexibility in an intermixed, task-switching context, characterized by the "all or none" principle where participants inhibit a dominant response all the time, was evident in participants with mature cognitive system, after 13 years of age. The last finding indicates that adult participants adjusted their speed to preserve accuracy on the more difficult trials. On the contrary, young children were often too impulsive to take the time they needed at the cost of accuracy. Moreover, adults seemed to be able to reset their default response between the two conditions of the Mixed block in order to exercise inhibition in a steady state. Furthermore, the study aimed to delineate the interactions among the basic executive processes, such as inhibitory control, memory and task switching. As far as the inhibitory control is concerned, the ability to inhibit attention to distractors or the ability to inhibit a strong behavioral inclination is tied to a person's cognitive flexibility. Consequently, selective and sustained attention is critical for adaptability and creativity in the way people hold and manipulate information. When the tasks were similar in difficulty, participants' speed on working memory and inhibition were highly correlated, whereas their accuracy on working memory and inhibition was respectably correlated. 
Anderson[9] conceptualizes executive function as an integrated supervisory or control system, made up of multiple process-related systems. More specifically, attentional control processes include the capacity to selectively attend to specific stimuli and inhibit prepotent responses as well as the ability to focus attention for a prolonged period. In advance, individuals' acquiring attentional control involves the regulation and monitoring of actions in order for them to complete tasks, avoid procedural mistakes and respond appropriately. Furthermore, information processing can be evaluated by the speed, quantity and quality of output. Cognitive flexibility, including the working memory capacity, refers to the ability to shift between response sets, learn from mistakes, devise alternative strategies, divide attention and process multiple sources of information concurrently. Finally, goal setting lays on individuals' ability to develop new initiatives and concepts while planning actions to approach tasks in an efficient and strategic manner. Effective assessment of children's executive function is quite challenging due to the need for capturing quantitative factors as well as cognitive-related processes and behavioral elements. Moreover, individuals' executive control reaches its maturity when the respective cognitive processes are fully established around mid-adolescence or early adulthood. Therefore, longitudinal studies will be required so as to verify any conceptualization concerning executive function development.

Keil et al.[10] describes executive processes as a prerequisite function for individuals' adequate response to novel and complex environmental demands. The former consist of several cognitive processes, such as self-monitoring, self-awareness and self-regulating, inhibiting irrelevant stimuli, shifting between concepts or actions, generation and application of strategies, temporal integration of information as well as recruiting or integrating multimodal inputs from throughout the brain. More specifically, researchers compiled an annotation of neuropsychological tests for the purpose of identifying the abilities that underlie executive functions and therefore discriminate cognitively impaired patients. Neuropsychological experiments should encompass multiple measures or administration of control tasks in addition to executive-level tasks so as to discern the executive functions from other contributing factors, such as basic or focal processes. Overall, control tasks should tap visuospatial, attentional or other processes likely to be involved in respective executive function tests. Moreover, the cognitive processes addressing participants' planning, scheduling, strategy use and rule adherence compose the first set of executive function tests. In addition, executive control processes, such as generating word or designs without repetition and complying with environmental constraints, are subject to monitoring skills and can be measured by fluency and generation tests. The third category of tests is centered on sustaining or/and shifting attention and suppression of habitual responses through self-regulating one's behavior. Finally, tests of concept formation and abstract reasoning, requiring continuous monitoring and modulation of output, tap another domain of executive functions. As many of the aforementioned tests deploy linguistic processes or language, the relation between executive functions and verbal intelligence via internal speech or verbal mediation in language-disordered population, should be clarified. 
Strobach et al.[11] showed that experienced video gamers acquire optimized and speeded executive control processes as they coordinated two different tasks either simultaneously in dual-task situations with novel stimuli or stimulus-response mappings or sequentially in task switching tests. The researchers made two experiments in order to investigate video game trainees' improved executive control skills in an attempt to select and execute multiple responses. The first experiment taken place, involved a dual-task test consisting of a single-task situation and a series of dual-task block trials with the simultaneous presentation of auditory and visual stimuli. The second one, constituted a task-switching test with single and mixed-task blocks, wherein predictive switches occurred between task-switch trials and repeat trials. Both tests were performed before and after video game practice. A scatter plot of the Brinley-plot type demonstrated action video gamers' superior executive control skills in complex situations calling for an effective regulation of the switch between conflicting stimuli in intermixed task settings. It seems that executive control skills can be improved, thus become traceable when a person coordinates different tasks involving simultaneous or sequential, but certainly rapid switches between them.

Pureza et al. [12] present the relationship between executive functions, such as the central executive of working memory, inhibition, cognitive flexibility and selfmonitoring in late childhood. The instruments deployed were the Unconstrained, Phonemic and Semantic Verbal Fluency subtests of the Montreal Battery of Evaluation of Communication, the Random-Number Generation task, the Bells Test, the n-back Test and the Hayling Test. The results suggest a relationship between inhibitory control, cognitive flexibility, processing speed as well as the central executive of working memory. More specifically, a strong association was brought into light between language alternation as an aspect of cognitive flexibility and inhibitory control as the ability to inhibit responses unrelated to the context. Likewise, selective attention and processing speed in verbal fluency were also related. On the one hand, attention, inhibition and verbal-semantic linguistic processing and on the other hand, focused attention, inhibition, verbal processing speed and self-monitoring were found to be linked. Furthermore, inhibition and cognitive flexibility of semantic verbal fluency were related to the central executive component of working memory. Regression analysis on larger samples could verify and expend current findings.

Rudkin et al.[13] inquired into the relation between the visual-spatial and central executive components of working memory through a triplet of dual-task coordination experiments. The central executive is responsible for complex functions, such as comprehension, reasoning, strategic cognitive control and dual-task coordination. Both visual and spatial working memory tasks, the Matrix Patterns and the Corsi Blocks respectively, were used as a means for discriminating the function of visual and spatial working memory subcomponents. The dynamic role of the spatial component is apparent as it records and encodes information about movements and sequences of movements as well as it entails one's inner ability to monitor and control his spatial attention. Thus, there seems to be an interrelation between one's executive functioning and his visual-spatial processes, which is worthy to be looked into. In the first experiment, central executive processes, such as information updating, attention shifting and inhibiting inappropriate information were more closely related to the 
spatial component than the visual component in a dual-task condition. In this case, both the Matrix Patterns task and the Corsi Blocks task were combined concurrently with an oral random generation task. Participants' performance in the spatial task decreased much more than their performance in the visual task, indicating the former's tapping of executive processes, although there was no significant difference in random generation performance between the two dual-task conditions. In the second experiment, researchers investigated the effect of a dual-task condition experiment combining the concurrent presentation of an oral random generation task and a visual-spatial task, either with sequential or simultaneous stimuli. The results of the second experiment verified the assumption that the recall of both item and order information in a serial sequential presentation taps of central executive functioning more than in simultaneous visual-spatial tasks. Finally, the third experiment controlled for the interference of short-memory in the random generation task, thereby minimizing its influence on the measurement of executive functioning. The two previous visual-spatial tasks were concurrently combined with either random- and fixed-interval auditory tone repetition tasks. More specifically, the fixed-interval tone task was expected to load the executive to a lesser extent than the random-interval task. However, both tone tasks interfered with the sequential presentation task only and the disruption caused by the two tone tasks was not significantly different. Overall, a serial-sequential visual-spatial task taps of executive processes to a greater extent than simultaneous item information presentation tasks. This is because sequential presentation may lead to a significant increase in one's demand of strategic monitoring and control over the encoding and rehearsal of the mental path configurations from the movement patterns of the stimuli. The question remains which part of the sequential task, encoding, maintenance or retrieval, places greater executive load.

Yates et al.[14] made a correlational study between executive processes and other functions, such as working memory, the executive attention construct, attention and prospective memory. In this study, it is insinuated that shifting, inhibition and updating are three independent, but interrelated subprocesses compiling one's executive function. Shifting corresponds to attention switching or task switching. Inhibition is the intentional and controlled suppression of dominant, automatic and prepotent responses. Updating lays on renewing and monitoring the working memory representations. Previous studies considered executive functions as a fraction of goal directed behavior, attention control, temporal organization and planning. In the current study, the Wisconsin Card Sorting Test was implemented as a means for measuring participants' cognitive flexibility and problem solving skills. In addition, the Brazilian Brief Neuropsychological Assessment NEUPSILIN measured participants' time and spatial orientation, sustained auditory attention, visual perception, memory, arithmetic abilities, language skills, motor abilities and two subcomponents of executive functions, such as solving simple problems and verbal phonemic-spelling fluency. The study aimed at relating one's performance in the WSCT to one's performance in the cognitive tasks encompassed in the NEUPSILIN. The results verify the existence of the executive attention construct that is the ability to keep an objective in active state during the task and the ability to overcome 
interference, particularly in a conflicting situation. Sustained attention, prospective memory and verbal fluency were moderately correlated in tests as well as executive functions correlated with episodic-semantic memory and oral and written language. Furthermore, one's executive function was related to one's working memory capacity. WCST entails both visual and oral language stimuli for the tapping of abstraction, insight formation and mental flexibility, thus depicting the multi-modality of the measure. Nevertheless, more implicit language recognition demands in the tasks would deliver greater language inferential processing associations by virtue of the close relation between visual-spatial information and executive functioning or attentional control.

Cinan et al.[15] shed light on the interplay between the central executive and the phonological loop through manipulating the Wisconsin Card Sorting Test. The executive systems responsible for the different types of executive functions involve the phonological loop and the visuospatial sketchpad. In the current study, a dual-task design was included, with the secondary tasks either taxing the phonological loop or the central executive so as to investigate on the specific role of the phonological loop while addressing the novel types of the WCST. In all three versions, the WCST-4 stimuli, the WCST-12-stimuli and the WCST-12-stimuli-box, participants were given the exact sorting criterion in order to avoid loading on the phonological memory. However, in the WCST-4 stimuli the same four stimulus card materials were used as in the original WCST. On the contrary, the WCST-12-stimuli and the WCST-12stimuli-box encompassed unidimensional stimulus cards. More specifically, in the WCST-12-stimuli-box condition, boxes were used to conceal the response cards previously sorted. In the first two dual-task experiments, the WCST-4 stimuli were performed simultaneously with either an articulatory suppression task or the letter generation task accompanied by a control, single-task condition. The articulatory suppression task taps the phonological loop and the letter generation task taps the central executive. The second two dual-task experiments included the concurrent use of the WCST-12-stimuli with the letter generation task as well as the use of the WCST-12-stimuli-box with the letter generation task. The study verified that the phonological secondary task had no effect on the performance of the simple version of the WCST, aimed to relish participants of any phonological memory load. Therefore, the response inhibition processes measured primarily in the simple new version of the WCST were found to be mediated by the executive secondary task, whereas participants' inhibitory control was found not to be mediated by the simultaneous use of their phonological loop. Moreover, the visual features taxing participants' inhibitory control in the simplest type of the WCST as well as participants' concurrent use of the executive secondary task affected their overall executive function. Researchers place their future interest on the separate role of allocating attention in the central executive, mainly through adopting a version of the WCST that entails updating and maintaining the information about the sorting criterion of 12 stimulus cards, mixed and placed in a random way. The response cards could be shown one by one by the researchers and the ones shown could be hidden. The participants should not be given the sorting criterion. Thus, the aforementioned experiment could offer the means for a thorough investigation on the role of attention 
and visual-spatial working memory in individuals' executive function as well on the possible relation between attention and visual-spatial working memory.

Nouchi et al.[16] made an intervention study on the effects of a Learning Therapy encompassing reading aloud and solving simple arithmetic calculations in elderly people on a range of cognitive processes, such as processing speed, verbal and facial episodic memory, short-term memory, working memory, focus attention, reading ability and inhibition and shifting as executive functions. The cognitive intervention had duration of 23 weeks, during which the difficulty level of training tasks and workloads did not change. Before and after the intervention period, the following cognitive measures were implemented: the Stroop Test and the Verbal fluency task measuring executive functions, the Digit cancellation task measuring attention, the Logical memory as well as the First and second names test measuring Episodic memory, the Digit span forward measuring Short-term memory, the Digit span backward measuring Working memory, the Japanese reading test measuring Reading ability and finally, the Digit symbol coding and the Symbol Search test measuring Processing speed. The cognitive intervention group exhibited improved inhibition, verbal episodic memory, focus attention and processing speed compared to the waiting list control group. The aforementioned relation was investigated by virtue of the overlapping cognitive processing underlying the learning therapy and the cognitive measures. For instance, comprehending and reading a sentence relies on semantic and verbal episodic memory as well as on focus attention. Processing speed and inhibition affects arithmetic calculation problem tasks. However, shifting and facial episodic memory measures remained constant as their enfolded cognitive processes, such as clustering, switching, face recognition and association were intact by the learning therapy. Therefore, executive functions and episodic memory as well as working memory are constructs that necessitate multiple measures for investigating individuals' cognitive functions. In addition, future studies should entail intensive adaptive training throughout the intervention period including other types of cognitive training, such as working memory training.

Fried et al.[17] made a comparative study concerning the effects of working memory deficits on children with and without ADHD. Working memory deficits have already been linked to academic deficits beyond ADHD. It is worth mentioning that one study showed the negative impact of impaired working memory on non-ADHD individuals' focusing attention, inhibition of irrelevant stimuli, recognition of priority patterns, ability to recognize hierarchies and the meaning of stimuli, establishing an intention as well as recognizing and selecting the goals that are best suited to solving a problem. In this study, the scope of impact of deficits in working memory was evaluated through stratifying children's with and without ADHD working memory deficits. The psychiatric assessment was succeeded by the intellectual functioning assessment encompassing the Vocabulary and Block Design, subtests of the Wechsler Intelligence Scale for Children-Revised as well as the Freedom from Distractibility (FFD) Factor from the WISC-R. The first tests measured participants' IQ and the second assessed the working memory. Reading and arithmetic achievement were measured with subtests of the Wide Range Achievement Test-Revised. The findings verified that working memory deficits in ADHD children significantly increased the 
risk for grade retention, placement in special classes and lower academic achievement, both in reading and math. More specifically, children with ADHD and comorbid working memory deficits are much more susceptible to cognitive and academic deficits in contrast to children with ADHD and no deficits in working memory. As working memory deficits and ADHD represent separate, comorbid conditions, appropriate educational intervention could assist children face up to their respective adversities. In addition, children with ADHD and with comorbid working memory deficits may comprise an innovative field of study in neuroscience. Research on the educational and cognitive outcomes in the general population originating from working memory deficits is also an ample field of study.

Francesco et al.[18] reviewed on the executive function deficits in AttentionDeficit/Hyperactivity Disorder (ADHD) and Autism Spectrum Disorder (ASD). Research on the executive function deficit directly associated with the aforementioned groups lays on the latter's shared genetic and neurobiological underpinnings, leading to a disturbance at the level of the executive functions. Moreover, the co-occurrence of ASD and ADHD could inaugurate a combined phenotype, reflecting an additive comorbidity, thus augmenting enhanced treatment options. Executive functions, such as planning, spatial and verbal working memory, response inhibition and vigilance have been linked to ADHD children/adolescents. Moreover, an ADHD subtype, the ADHD-I has been related to sluggish, disorganized behavior. Children with ADHD also have deficits in sustained attention and visual memory. Likewise, constant impairments in cognitive flexibility, planning and working memory have been found in individuals with high-functioning autism. In addition, children with autism have shown more deficits in shifting attention, sustained or selective attention and response inhibition. Furthermore, a clinical combined phenotype, ASD+ADHD, was attempted to be investigated separately regarding the level of the following executive functions: inhibition, working memory, flexibility, attention, planning, monitoring, preparatory processing, fluency and concept formation. Attention was found to be dysfunctional in all clinical groups (ASD, ADHD and ASD+ADHD). The ADHD children had a deficit in the manipulation of sustained and divided attention compared to healthy children, whereas children with ASD+ADHD performed worse in divided attention and alertness tasks compared to their healthy counterparts. In contrast, both ASD and ASD+ADHD groups were found to be lacking in cognitive flexibility and planning. The ASD was particularly related to cognitive flexibility deficit and the ASD+ADHD group was found to be impaired in duration concerning both flexibility and planning tests. ADHD and ASD+ADHD groups were also lacking in response inhibition. Consequently, cognitive training in executive functions may change the underlying neural mechanisms to improve the above groups' real-world function.

Mertes et al.[19] investigated the effects of spatially and temporally irrelevant information on the processing of subsequent information. Relevant information is maintained in visual working memory and its representations facilitate the adaptability of the human behavior. Notwithstanding, visual attention is captured by irrelevant objects in the visual field. Thus, these automatic, involuntary shifts of attention occur due to distracting salient stimuli or stimuli that match the representations held in visual working memory. As a result, suppression mechanisms 
are activated, the attentional control sets, to re-orientate and reallocate one's attention to the sought-after objects. Moreover, it has been found that individuals' responses at spatial cueing tasks are faster when the target and the preceding cue shared the same location. Attention was already drawn to the location of the cue, thereby reinforcing the processing of the object, subsequently presented at that location. In the present study, an irrelevant color cue that was either contingent (color search) or noncontingent (shape search) on attentional control sets was presented, prior to a target array with different stimulus-onset asynchronies (SOA). More specifically, cue displays containing a lateralized color singleton were presented prior to the target display. Participants were instructed to ignore the task-irrelevant cue display as the cue position did not allow for predicting the target position. In one condition, both cue and target singletons were defined by color (contingent condition). In the noncontingent condition, color singleton cue and target singleton did not share features in order to facilitate the inhibition of the color singleton cue. Researchers measured event-related potentials (ERPs) evoked by the cue display. The findings of the study sum up to two independent filtering stages regarding the passage of information into working memory. First, an early selection process represented by an N2 posterior contralateral (N2pc) to the singleton cue and a component labeled distractor positivity (Pd-early), enables the active re-orienting of the attentional focus in the search array when irrelevant information matches working memory templates. Second, there is a more conservative filtering stage determining visuospatial working memory access, associated with Contralateral delay activity (CDA) and distractor positivity (Pd-late) component, which encompasses the active maintenance of working memory representations surviving after the first inhibition of the cued location as well as the final inhibition of the false representations as soon as the relevant information is obtained. At that point individuals are able to proceed with the rapid perception of the target. Therefore, attentional processes seem to be strongly linked to cognitive control mechanisms, such as the distractor positivity $(\mathrm{Pd})$ component that assists people to prioritize their targets. The distractor positivity $(\mathrm{Pd})$ component has been found to be subjected to exterior incentives, such as rewards. Furthermore, individuals' attentional mechanisms through the manipulation of their distractor positivity $(\mathrm{Pd})$ component are adaptable and susceptible to motivation.

\section{Emotional / Motivational Executive Function}

Channon [20] describes brain-injured individuals' effect on their problem solving skills. More specifically, executive dysfunction in cognitively impaired individuals lays on their difficulty to shift between novel tasks due to their inability to access and manipulate prior knowledge, to appreciate the problem situation in working memory, to generate appropriate strategies, to inhibit inappropriate responses as well as to monitor and judge the effectiveness of problem solutions. Therefore, focus on individuals' actual behavior entails both their real-life-type problems and their actual problem-solving performance. Adults with focal lesions or other neurodevelopmental disorders, such as the Asperger's syndrome, Tourette's syndrome as well as healthy 
versus younger people were tested on their ability to generate as many possible ways of solving an awkward problem situation as they could. Each solution was further coded and rated in terms of the examinee's appreciation of the pertinent aspects of the problem, the social appropriateness of his solutions and the effectiveness of his solution from a practical viewpoint. After the solution generation phase, participants were asked to give their preferred final solution for the problem, both from the perspective of the main character and for themselves. Patients with left anterior lesions and the two neurodevelopmental groups showed impaired problem-solving, both in generating a range of high quality potential problem solutions as well as in selecting appropriate preferred solutions. In advance, the aforementioned groups' performances on clinical executive tests, such as the Wisconsin card sorting test, the Rule shift test, the Hayling test, the Letter fluency Test and the six Elements test were rather inconclusive as to the detection of differences between the groups. Nevertheless, there were positive correlations between the clinical executive tasks and the number of solutions generated as well as the average total final quality of solutions provided by the participants. Focusing and sustaining attention, generating and using an appropriate strategy, maintaining information in working memory, shifting between ideas and inhibiting inappropriate ideas is executive processes needed in everyday settings. Thus, weak performances on the average number of solutions or on selecting appropriate problem solutions result from deficiencies in abstract executive skills, such as encoding of successful methods of approaching problem situations and discarding or appropriate labeling of less successful methods were linked to a positive final outcome. In short, both quality of the available knowledge itself in combination with the identification, accessibility and activation of the necessary material in working memory form an efficient strategic memory operation. Nevertheless, life experience seems to be equally important in ill-defined problems as healthy older people showed preservation of performance on the complex problem solving task but performed worse on several of the clinical executive measures compared to the healthy young group. On the other hand, the Asperger syndrome group had a difficulty in selecting solutions that were socially appropriate, probably due to a deprived social life and thus, these people lacked in quality and quantity of social knowledge structure. Moreover, even though there is some evidence for the independence of social cognition and non-social executive abilities, the exact relation between executive functions and theory of mind skills seems to be rather vague.

Beck et al. [21] attempted to link middle-childhood children's personal characteristics to their innovative problem solving capacity. At first, researchers compared children's performance on tool-innovation tasks with measures of children's fluency in divergent thinking. In the Circles task, children drew as many different pictures as they could on a circle template, in the Object uses task, they had to suggest multiple uses for objects and in the Hook-innovation task the experimenter showed the child a bucket with a sticker at the bottom of the tall transparent tube and prompted the child to get the sticker. Notwithstanding, none of these measures of divergent thinking was a significant predictor of children's innovation success. Furthermore, children's fundamental executive functions, their inhibitory control, 
working memory and attentional flexibility were measured accompanied by the toolinnovation task; the Six Parts Test, a measure of ill structured problem solving. Additionally, children completed the British Picture Vocabulary Scale-II, a supplementary measure of general intelligence. The findings showed that there was a relationship between innovation and receptive vocabulary but no evidence between executive function and children's innovation success. The inconclusive results insinuate that it may be another cognitive process related to innovation, such as analogical reasoning as well as a personality trait and/or positive, social learning experiences of successful innovation that comprise an innovative child.

Petrovic et al. [22] presented an integrated model of mechanistically related processes, either emotional or non-emotional, tied to the dysfunctional top-down regulation of information processing in ADHD and emotional instability disorders. Top-down regulation refers to the reciprocal interaction of information between multiple regions in large-scale, emotional and non-emotional networks with hierarchical perception-action organization. Thus, the highest level of emotional and non-emotional processes performs the more complex and temporally dispersed information. Moreover, top-down control of the emotional and the non-emotional systems vary in their efficiency among the general population. In addition, ADHD patients have been associated with poor non-emotional top-down control and individuals with emotional instability disorders have been associated with poor emotional top-down control. Therefore, patients' with ADHD would show a dysregulation in the non-emotional system and normal emotional regulation, whereas individuals with emotional instability disorders would show a dysregulation in the emotional system and normal non-emotional regulation. Comorbid states may encompass poor capacity for both non-emotional and emotional processing. However, the aforementioned dysregulation shares common grounds on account of an underlying neuromodulatory mechanism, the dopamine system, which interacts with both emotional and non-emotional networks. Its proper treatment would affect the whole regulatory brain network, processing either emotional or non-emotional types of information.

Jiang et al.[23] explored emotional regulation and executive functions in the Oppositional Defiant Disorder (ODD) and in ADHD children. Additionally, the relationship between emotional regulation and executive functions were intrinsically investigated. In the dual-pathway model of executive functions, attention, working memory, planning and response inhibition pertain to cold executive functions in comparison with the hot executive functions embracing one's emotional and motivational regulation. Although ADHD children have been related to most executive function deficits, both cold and hot, ODD children have been connected mainly to deficits in individuals' visual working memory and response suppression/inhibition. The present study is particularly interested in the relation of ODD and ADHD children with emotional regulation as part of executive functioning, subjected to motivations and emotions. The Adolescent Daily Emotional Regulation Questionnaire (ADERQ), the Wisconsin Card Sorting Test (WCST), the Wechsler Intelligence Scale for Chinese children (WISC-IV), the Stroop color-word association test and the Cambridge Neuropsychological Test Automated Battery (CANTAB) 
were the research tools deployed. The findings of the study verified that ODD children face deficits in emotion regulation processes, albeit their exact behavioral characteristics as well as their whole executive functioning profile and the role of the comorbidity with the ADHD syndrome in their emotion dysregulation remains unclear. Notwithstanding, the ability to regulate emotions is an important part of an individual's executive functions. In this case, ODD children were prone to greater number of perseverative errors due to their lack in self-reflection, self-control and self-regulation of negative emotions combined with deficits in working memory and planning capabilities. On the whole, response inhibition incapability, planning dysfunction and emotion dysregulation were important risk factors for ODD children.

Van Stralen[24] made a review study on the relation between children's emotional dysregulation and attention-deficit/hyperactivity disorder. Emotional dysregulation stands for one's reactive or motivational control problems leading to hyperactivityimpulsivity symptoms due to inappropriate internalized (sadness, depression) or externalized (anger, aggressiveness) emotional responses. More specifically, the emotional dysregulation in ADHD refers to individuals' lack in a whole set of cognitive and meta-cognitive skills, such as attention, self-control, self-regulation and self-monitoring. Furthermore, emotional impulsiveness as well as deficient selfregulation of affections-motivation-arousal, internalization of speech and reconstitution in the ADHD syndrome co-occurs with other executive functions, such as inhibition and working memory. Latter research illustrated that ADHD individuals' behavioral dysregulation and difficulties in metacognition are interconnected. Thus, their emotional dysregulation and deficits in executive functions can be interdependent and all contribute independently to distinguishing between children with ADHD and controls. Further research on the field of emotional dysregulation in ADHD individuals could offer a crystallized definition of the construct as well as a comprehensive methodology for building innovative training and assessment tools.

\section{$4 \quad$ Executive Function Abilities and Problem Solving}

Nguyen et al. [25] made an intervention study to enhance older adults' complex decision-making abilities through a problem-solving therapy modality. It has been shown that some older adults may experience a greater decline in non-memory-related cognitive functioning, such as problem-solving and mental flexibility, contributing to weaknesses in their decision-making abilities [26]. The findings from the current study demonstrated that a four-session (approximately 2 weeks) problem-solving therapy requiring abstract problem-solving with inductive reasoning and flexible adjustment of responses based on feedback can reinforce aspects of executive functioning (that may have declined as a part of healthy aging), thereby enhancing decision-making abilities. Such executive skills encompass emotional regulation, behavioral initiation, planning, organization, cognitive flexibility and problem-solving [27]. In conclusion, this intervention promoted older adults' self-efficacy and ability towards resolving practical everyday challenges. 
Problem solving therapy (PST) relies on a learning model that assists older adults with major depression and executive dysfunction to identify problems central to their lives, select solutions and make concrete plans for problem resolution. This therapy has been found effective for improving patients' self-care, household and work activities, getting around, understanding and communicating, getting along with others as well as participating in social activities [28].

Kurowski et al.[29] described their randomized clinical trial (RCT) design to examine the comparative effectiveness of three versions of F-PST (family problemsolving therapy) in improving/ameliorating patient- and caregiver-reported behavioral outcomes regarding traumatic brain injury (TBI). Face-to-face; online and selfdirected and therapist-supported online modes of treatment are going to be tested for their effectiveness in patient outcomes. Youth with TBI and their families in all three treatment groups will receive 10 sequential sessions providing training in staying positive/cognitive reframing, problem-solving, communication, and selfregulation/anger management. Parents and youth with TBI will complete the Behavior Rating Inventory of Executive Function (BRIEF), a rating of the child's executive functioning abilities.

Zelazo et al. [30] aimed to improve preschool children's executive function skills, such as cognitive flexibility, working memory and inhibitory control through goaldirected problem solving. As it is already known executive function skills can be fostered through reflection training as individuals acknowledge and are able to manipulate their cognition and emotions. The 6-week intervention comprising both mindfulness and reflection training was found to improve preschool children's executive function skills in the long run, which indicates a transfer effect of the trained executive skills on future problem-solving situations and assessments [31].

Problem-centered, collaborative environments offer personal challenges and, over the long run, help students gain higher-order executive processes. Effective instructing is a dynamic, interactive process, akin to problem-solving [32]. Executive functioning is the most important process for adaptive, planful learning and thinking. Initially, the primary executive process is to analyze the task at hand and to select an appropriate strategy; during the course of learning, its role shifts to strategy monitoring and revision [33]. Interestingly, in younger children, inhibition was found to be the strongest predictor of problem solving, whereas working memory contributed more strongly in older children's problem solving capacity [34]. In another study, cool Executive Functioning (EF) predicted math achievement, learning-related classroom behaviors and observed engagement in kindergarten [35].

In addition, research suggests a vital role for executive function in children's social-emotional development. However, executive function is rarely considered in models of intervention programs that attempt to promote social-emotional competence [36]. Children who demonstrated better inhibitory control were more likely to be rated higher on social skills and lower in internalizing behaviors. Findings suggest that early identification of inhibitory control difficulties may be beneficial for targeting children at risk for maladaptive outcomes. Moreover, executive function (EF) in 6 year old children predicted first-grade teachers' ratings of emotional symptoms, hyperactivity, and conduct/peer problems [37]. The contribution of 
environmental experience to the development of inhibitory control skills suggests there are many opportunities to intervene during early childhood [38].

The neuropsychological symptoms seen in adults with ADHD may be explained by deficits in executive function, mainly composed of inhibition and metacognition. Deficits in inhibition of motor, verbal, cognitive, and emotional activities contribute to inattention in adults with ADHD. These deficits originate in metacognitive aspects of cognition, such as nonverbal working memory, verbal working memory, planning and problem-solving, as well as emotional self-regulation. Therefore, by enhancing metacognitive functions, inhibitory control could also be improved [39].

Furthermore, Goal Management Training is a metacognitive, tailor-made intervention for executive functioning deficits intended to promote a mindful approach to problem-solving by raising awareness of attentional lapses and reinstating cognitive control when behavior is mismatched to the ongoing goal hierarchy [40, 41, 42]. In addition, executive functions receive input from higher-level metacognitive processes, such as self-monitoring, self-assessment, values, motivations and beliefs about the "self.". This active self-monitoring may facilitate the revision or updating of the individual's metacognitive beliefs, but also contributes to making decisions about the use of strategies, called self-control [43].

Therefore, disorders of planning and problem solving, such as Traumatic Brain Injury (TBI) can be treated through improving individuals' self-monitoring accuracy and thus, strategic decision-making [43]. In addition, the maturity of social problemsolving skills mediates the relationship between executive function and social outcome in paediatric traumatic brain injury (TBI) [44].

\section{$5 \quad$ Results}

The common ground of the aforementioned studies lays on the distinct, although interdependent, executive functions and their relation to individuals' everyday behavior and learning. Either intellectually-based processes or emotional/motivational-based executive processes, they are all necessary for every day, complex problem solving tasks [48]. Therefore, executive functions consist in cognitive, metacognitive and emotional constructs, especially individuals' sustained or selective attention, response inhibition and emotional regulation. In advance, executive functions are innately related to encoding, accessing, updating and selfmonitoring of problem-solving strategies and solutions, such as those learned in science, maths and technology [49].

Moreover, it is hypothesized that attention and the cognitive control mechanism could have a positive effect on the metacognitive, regulatory mechanism behind the emotional system, as well. In any case, the attentional control mechanism was preliminary destined to improve infants' behavior. Therefore, an improvement in the attentional control mechanism could offer either an emotional boost or an adaptable behavior, possibly due to one's capacity to regulate his emotions.

By being able to self-regulate cognition and emotion in a systemic way, individuals could improve their actual problem solving performance and real-world function. 
More specifically, several everyday decision-making issues require shifting between novel tasks, appreciating the problem situation according to the representations located in the working memory, generating appropriate problem-solving strategies, inhibiting inappropriate responses as well as monitoring and judging the effectiveness of problem solutions. Furthermore, emotional regulation as the counterpart of metacognitive executive functions influences individuals' social behavior, an intrinsic human need.

Attention skills were found to be related to inhibitory control so as to support individuals' cognitive flexibility; that is the ability to inhibit attention to distractors or the ability to inhibit a strong behavioral inclination. Thus, selective and sustained attention has a key role in the way people hold and manipulate information. Therefore, attentional control processes encompass the capacity to selectively attend to specific stimuli, inhibit prepotent responses as well as the ability to focus attention for a prolonged period and regulate/monitor one's actions.

In addition, according to Ardila[2], individuals' attentional control processes are primary (they emerge by the child's 1st year), include meta-cognitive control mechanisms and lead to improvements in the ability to inhibit overlearned behavior (inhibitory control of behavior). Furthermore, attention was found to be adaptable and subjected to affective states (motivation). Controlling emotions and the brain's reward systems are also intrinsically related processes [45]. Therefore, attentional control and emotional regulation have a common mediator; motivation. Overall, by improving executive functions and especially individuals' attentional control, their learning capacity and social adaptability is enhanced through their cognitive and emotional upgrade.

\section{Conclusion}

Executive functions are interweaved cognitive and metacognitive processes encompassing individuals' cognitive skills, values, beliefs as well as self-regulation abilities. Self-regulation regarding one's cognitive skills, such as attention, memory, inhibitory control, problem solving skills has proven to be related to one's emotional self-regulation. Thus, individuals facing emotional and/or executive shortcomings, such as older adults, individuals facing Traumatic Brain Injury (TBI), individuals with ADHD, Autism Spectrum Disorder as well as Oppositional Defiant Disorder and their comorbidities can be substantially assisted in their everyday problem solving through improving their overall self-regulation. Moreover, by training young children's selfregulation, their learning potential and conduct are improved as their cognitive/metacognitive and emotional processes are enhanced.

More specifically, metacognitive executive functions are complementary to each other as attention, self-monitoring, working memory capacity, inhibitory control and task switching are interrelated functions, affecting individuals' cognitive control mechanism. Even more, attention seems to have a supreme role among these core, metacognitive executive functions in the orchestration of information processing, thus creating cognitive flexible individuals. However, attentional processes develop early 
in humans in order to address their need for adapting their behavior. In this sense, attentional control could improve individuals' emotional regulation throughout their growth, as well.

In essence, attentional control can facilitate individuals' whole spectrum of executive functioning, cognition and behavior. Interestingly, selective and sustained visual attention measured through a distractor positivity $(\mathrm{Pd})$ component in a serial sequential presentation attentional control, were found to be susceptible to exterior incentives. Thus, attention processes are adaptable and liable to training [46, 47].

Consequently, future studies should look into the relation between attentional processes and one's ability to regulate his emotional/motivational executive functioning; One's emotional/motivational executive functioning is tied to the underlying cognitive control processes regarding his inner and social emotions. In that case, older adults, individuals facing Traumatic Brain Injury (TBI), individuals with ADHD, Autism Spectrum Disorder as well as Oppositional Defiant Disorder and their comorbidities, would be sufficiently assisted in their need to regulate their emotions or motivational control. Such an advanced research in the bidirectional relation between executive function and problem solving skills could develop a comprehensive methodology for training and assessing self-regulatory processes.

Moreover, the aforementioned research would offer tangible proof of the fact that metacognitive executive functions and emotional/motivational executive functions are interweaved in a holistic view of "self-consciousness". That is the interdependence of one's cognition/metacognition and his inner emotions or even further, the interdependence of one's cognition/metacognition and his social emotion (theory of mind).

\section{$7 \quad$ References}

[1] Ardila, A. (2016). Is self-consciousness equivalent to executive function? Psychology and Neuroscience, 1-6. https://doi.org/10.1007/978-3-319-50340-0 43

[2] Ardila, A. (2013). Development of Metacognitive and Emotional Executive Functions in Children. Applied Neuropsychology: Child, 1-6. https://doi.org/10.1 $\underline{080 / 21622965.2013 .748388}$

[3] Fischer, A. and Neubert, J. C. (2015). The multiple faces of complex problems: A model of problem solving competency and its implications for training and assessment. Journal of dynamic decision making, 1(6): 1-13. https://doi.org/10.3991/ijep.v8i2.8228

[4] Rhodes, M. G. and Kelley, C. M. (2005). Executive processes, memory accuracy, and memory monitoring: An aging and individual difference analysis. Journal of Memory and Language, 52: 578-594. https://doi.org/10.1016/j.jml.2005.01.014

[5] Carden, J. and Cline, T. (2015). Problem solving in mathematics: the significance of visualization and related working memory". Educational Psychology in Practice, 31(3): 235-246. https://doi.org/10.1080/02667363.2015.1051660

[6] Passolunghi, C. M. (2001). Short-Term Memory, Working Memory, and Inhibitory Control in Children with Difficulties in Arithmetic Problem Solving. Journal of Experimental Child Psychology, 80: 44-57. https://doi.org/10.1006/jecp.2000.2626 
[7] Zheng, X. Swanson, H. L. and Marcoulides, G. A. (2011). Working memory components as predictor of children's mathematical word problem solving. Journal of Experimental Child Psychology, 110: 481-498. https://doi.org/10.1016/j.jecp.2011.06.001

[8] Davidson, M. C. Amso, D. Anderson, L. C. and Diamond, A. (2006). Development of cognitive control and executive functions from 4 to 13 years: Evidence from manipulations of memory, inhibition, and task switching. Neuropsychologia, 44 (11): 2037-2078. https://doi.org/10.1016/j.neuropsychologia.2006.02.006

[9] Anderson, P. (2002). Assessment and Development of Executive Function (EF) during Childhood. Child Neuropsychology, 8(2): 71-82. https://doi.org/10.1076/chin.8.2.71.8724

[10] Keil, K. and Kaszniak, A. W. (2002). Examining executive function in individuals with brain injury: A review. Aphasiology, 16(3): 305-335. https://doi.org/10.1080/ $\underline{02687030143000654}$

[11] Strobach, T. Frensch, P. A. and Schubert, T. (2012). Video game practice optimizes executive control skills in dual-task and task switching situations. Acta Psychologica, 140: 13-24. https://doi.org/10.1016/j.actpsy.2012.02.001

[12] Pureza, J. R. Jacobsen, G. M. Oliveira, R. G. and Fonseca, R. P. (2011). Relationships between executive function tasks in late childhood. Psychology \& Neuroscience, 4(3): 369-376. https://doi.org/10.3922/j.psns.2011.3.010

[13] Rudkin, S. J. Pearson, D. G. and Logie, R. H. (2007). Executive processes in visual and spatial working memory tasks. The Quarterly Journal of Experimental Psychology, 60( 1): 79-100. https://doi.org/10.1080/17470210600587976

[14] Yates, D. B. Zibetti, M. R. Pawlowski, J. Salles, J. F. De Mattos Pimenta Parente, M. A. De Lima Argimon I. Fonseca, R. P. and Trentini, C. M. (2013). WCST and NEUPSILIN: Relationships among Executive Functions, Attention, Memory and Language. Psicologia: Reflexao e Critica, 26(3): 506-515. https://doi.org/10.1590/s0102-79722013000300010

[15] Cinan, S. and Tanor, O. O. (2002). An attempt to discriminate different types of executive functions in the Wisconsin Card Sorting Test. Memory, 10(4): 277-289. https://doi.org/10.1080/09658210143000399

[16] Nouchi, R. Taki, Y. Takeuchi, H. Nozawa, T. Sekiguchi, A. and Kawashima, R. (2016). Reading aloud and Solving simple arithmetic calculation intervention (Learning Therapy) improves inhibition, verbal episodic memory, focus attention and processing speed in healthy elderly people: Evidence from a randomized controlled trial. Frontiers in Human Neuroscience, 10(217): 1-14. https://doi.org/10.3389/fnhum.2016.00217

[17] Fried, R. Chan, J. Feinberg, L. Pope, A. Woodworth, K. Y. Faraone, S. V. and Biederman, J. (2016). Clinical correlates of working memory deficits in youth with and without ADHD: A controlled study. Journal of Clinical and Experimental Neuropsychology, 38(5): 487-496. https://doi.org/10.1080/13803395.2015.1127896

[18] Francesco, C. Francesco, M. Legrottaglie, A. R. Palumbi, R. De Giambattista, C. and Margari, L. (2016). A review of executive function deficits in autism spectrum disorder and attention-deficit/hyperactivity disorder. Neuropsychiatric Disease and Treatment, 12: 1191-1202. https://doi.org/10.2147/ndt.s104620

[19] Mertes, C. Wascher, E. and Schneider, D. (2016). From capture to inhibition: How does irrelevant information influence visual search? Evidence from a spatial cuing paradigm. Frontiers in Human Neuroscience, 10(232): 1-13. https://doi.org/10.3 389/fnhum.2016.00232

[20] Channon, S. (2004). Frontal lobe dysfunction and everyday problem solving: Social and non-social contributions. Acta Psychologica, 115: 235-254. https://doi.org/10.10 $\underline{16 / j . a c t p s y .2003 .12 .008}$ 
[21] Beck, S. R. Williams, C. Cutting, N. Apperly, I. A. and Chappell, J. (2016). Individual differences in children's innovative problem-solving are not predicted by divergent thinking or executive functions. Philosophical Transactions Royal Society B, 371: 1-11. https://doi.org/10.1098/rstb.2015.0190

[22] Petrovic, P. and Castellanos, F. X. (2016). Top-down dysregulation-From ADHD to Emotional Instability. Frontiers in Human Neuroscience, 10(70): 1-25. https://doi.org/10.3389/fnbeh.2016.00070

[23] Jiang, W. Li, Y. Du, Y. and Fan, J. (2016). Emotional regulation and executive function deficits in unmedicated Chinese children with Oppositional Defiant Disorder. Psychiatry Investigation, 13(3): 277-287. https://doi.org/10.4306/pi.2016.13.3.277

[24] Van Stralen J. (2016). Emotional dysregulation in children with attentiondeficit/hyperactivity disorder. ADHD Attention Deficit Hyperactivity Disorder, 1-13. https://doi.org/10.1007/s12402-016-0199-0

[25] Nguyen CM, Chen K-H and Denburg NL. (2018). The Use of Problem-Solving Therapy for Primary Care to Enhance Complex Decision-Making in Healthy Community-Dwelling Older Adults. Front. Psychol., 9(870) https://doi.org/10.3389/fpsyg.2018.00870

[26] Denburg, N. L., and Hedgcock, W. M. (2015). Age-associated executive dysfunction, the prefrontal cortex, and complex decision making, in Aging and Decision Making: Empirical and Applied Perspectives, eds T. M. Hess, J. Strough, and C. E. Lockenhoff. New York, NY: Academic Press, 81-104. https://doi.org/10.1016/b978-0-12-417148-0.00005-4

[27] Lezak, M. D., Howieson, D. B., Bigler, E. D., and Tranel, D. (2012). Neuropsychological Assessment, 5th Edn. New York, NY: Oxford University Press.

[28] Alexopoulos G. S., Raue, P.J., Kiosses, D.N. et al. (2011). Problem-Solving Therapy and Supportive Therapy in Older Adults with Major Depression and Executive Dysfunction. Arch Gen Psychiatry, 68(1): 33-41 https://doi.org/10.1001/archgenpsychiatry.2010.177

[29] Kurowski B.G., Stancin T. Taylor H.G. McNally K.A. Kirkwood M.W. Cassedy A. King E. Skluta M. Narad M.E. and Wade S.L. (2018). Comparative effectiveness of family problem-solving therapy (F-PST) for adolescents after traumatic brain injury: Protocol for a randomized, multicenter, clinical trial. Contemporary Clinical Trials Communications, 10: 111-120. https://doi.org/10.1016/j.conctc.2018.04.001

[30] Zelazo PD, Forston JL, Masten AS and Carlson SM. (2018). Mindfulness Plus Reflection Training: Effects on Executive Function in Early Childhood. Front. Psychol., 9(208) https://doi.org/10.3389/fpsyg.2018.00208

[31] Espinet, S. D., Anderson, J. E., and Zelazo, P. D. (2013). Reflection training improves executive function in preschool children: behavioral and neural effects. Dev. Cogn. Neurosci., 4: 3-15 https://doi.org/10.1016/j.den.2012.11.009

[32] Wood, D. and Middleton, D. (1975). A study of assisted problem-solving. British Journal of Psychology, 66: 181-191 https://doi.org/10.1111/j.2044-8295.1975.tb01454.x

[33] Borkowski, J. G., Chan, L.K.S. \& Muthukrishna, N. (2000). A Process-Oriented Model of Metacognition: Links between Motivation and Executive Functioning. Issues in the Measurement of Metacognition, ed. Gregory Schraw \& James C. Impara ,Lincoln, NE: Buros Institute of Mental Measurements.

[34] Senn, T.E. Andrews, K. Kaufmann, E. \& P. (2004). Using Path Analysis to Understand Executive Function Organization in Preschool Children, Developmental Neuropsychology, 26(1): 445-464 https://doi.org/10.1207/s15326942dn2601 5

[35] Brock, L. Rimm-Kaufman, S.E. Nathanson, L. \& Grimm, K.J. (2009). The contributions of 'hot' and 'cool' executive function to children's academic achievement, learning-related behaviors, and engagement in kindergarten, Early Childhood Research Quarterly, 24(3): 337-349, ISSN 0885-2006 https://doi.org/10.1016/j.ecresq.2009.06.001 
[36] Riggs, N.R. Jahromi, L.B. Razza, R.P. Dillworth-Bart, J.E. \& Mueller, U. (2006). Executive function and the promotion of social-emotional competence, Journal of Applied Developmental Psychology, 27(4): 300-309 https://doi.org/10.1016/j.appdev.2006.04.002

[37] Hughes, C. \& Ensor, R. (2011). Individual differences in growth in executive function across the transition to school predict externalizing and internalizing behaviors and selfperceived academic success at 6 years of age, Journal of Experimental Child Psychology, 108(3): 663-676, ISSN 0022-0965 https://doi.org/10.1016/j.jecp.2010.06.005

[38] Rhoades, B.L. Greenberg, M.T. \& Domitrovich, C.E. (2009). The contribution of inhibitory control to preschoolers' social-emotional competence, Journal of Applied Developmental Psychology, 30(3): 310-320 https://doi.org/10.1016/j.appdev.2008.12.012

[39] Barkley, R.A. (2010). Differential Diagnosis of Adults With ADHD: The Role of Executive Function and Self-Regulation. Supported by an educational grant from Eli Lilly and Company.

[40] Robertson, I. H. (1996). Goal Management Training: A Clinical Manual. Cambridge: PsyConsult.

[41] Levine, B., Stuss, D. T., Winocur, G., Binns, M. A., Fahy, L., Mandic, M., Bridges, K., and Robertson, I. H. (2007). Cognitive rehabilitation in the elderly: effects on strategic behavior in relation to goal management. J. Int. Neuropsychol. Soc., 13: 143-152. https://doi.org/10.1017/s1355617707070178

[42] Levine, B. Schweizer, TA. O'Connor, C. Turner, G. Gillingham, S. Stuss, DT. Manly T. and Robertson, IH. (2011). Rehabilitation of executive functioning in patients with frontal lobe brain damage with goal management training. Frontiers in Human Neuroscience, 5(9): 1-9 https://doi.org/10.3389/fnhum.2011.00009

[43] Kennedy M.R.T. \& Coelho C. (2005). Self-Regulation after Traumatic Brain Injury: A Framework for Intervention of Memory and Problem Solving. Seminars in Speech and Language, 26(4). https://doi.org/10.1055/s-2005-922103

[44] Muscara, F., Catroppa, C. and Anderson, V. (2008). Social problem-solving skills as a mediator between executive function and long-term social outcome following paediatric traumatic brain injury. Journal of Neuropsychology, 2: 445-461, https://doi.org/10.1348/174866407x250820

[45] Skogli, E. W. Egeland, J. Andersen, P. N. Hovik, K. T. and Øie, M. (2013). Few differences in hot and cold executive functions in children and adolescents with combined and inattentive subtypes of ADHD. Child Neuropsychology: A Journal on Normal and Abnormal Development in Childhood and Adolescence, 1-23. https://doi.org/10.1080 /09297049.2012.753998

[46] Zendel, B. R. De Boysson C. Mellah S. Demonet J.-F. and Belleville S. (2016). The impact of attentional training on event-related potentials in older adults. Neurobiology of Aging, 47: 10-22. https://doi.org/10.1016/j.neurobiolaging.2016.06.023

[47] Sperduti, M. Makowski, D. and Piolino, P. (2016). The protective role of long-term meditation on the decline of the executive component of attention in aging: a preliminary cross-sectional study. Aging, Neuropsychology and Cognition, 23(6): 691-702. https://doi.org/10.1080/13825585.2016.1159652.

[48] Wedelin, D and Adawi, T. (2014).Teaching Mathematical Modelling and Problem Solving - A Cognitive Apprenticeship Approach to Mathematics and Engineering Education. iJEP, 4(5), Special Issue: "CISPEE". https://doi.org/10.3991/ijep.v4i5.3555

[49] Zygouris, N.C. Vlachos, F. Dadaliaris, A.N. Oikonomou, P. Stamoulis, G.I. Vavougios, D. Nerantzaki, E. and Striftou, A. (2017). A Neuropsychological Approach of Developmental Dyscalculia and a Screening Test Via a Web Application. iJEP, 7( 4). https://doi.org/10.3991/ijep.v7i4.7434 
[50] Drigas, A. Karyotaki, M. and Skianis Ch. (2018). Mindfulness Training, Assessment and Intelligence. iJES, 6(3).

[51] Drigas, A. Karyotaki, M. and Skianis Ch. (2018). An Integrated Approach to Neurodevelopment Neuroplasticity and Cognitive improvement, iJES, 6(3). https://doi.org/10.3991/ijes.v6i3.9034

[52] Drigas, A. Skianis Ch. and Karyotaki, M. (2017). Success: A 9 Layered-based Model of Giftedness, iJES, 5(4). https://doi.org/10.3991/ijes.v5i4.7725

\section{Authors}

A. Drigas is with N.C.S.R. 'Demokritos', Institute of Informatics and Telecommunications, and Coordinator of Net Media Lab \& Brain-Mind R\&D, Agia Paraskevi, 153 10, Athens, Greece (e-mail: dr@iit.demokritos.gr).

M. Karyotaki is with N.C.S.R. 'Demokritos', Institute of Informatics and Telecommunications, Net Media Lab \& Brain-Mind R\&D, Agia Paraskevi, 153 10, Athens, Greece (e-mail: karyotakimaria@gmail.com).

Article submitted 2019-01-21. Resubmitted 2019-04-18. Final acceptance 2019-04-27. Final version published as submitted by the authors. 